\title{
Grado de satisfacción e impacto social y medioambiental en pacientes incluidos en un programa de seguimiento remoto de marcapasos
}

\author{
Degree of satisfaction and social and environmental impact in patients included in a \\ remote pacemaker monitoring program
}

\author{
Pedro Pérez-Díaz*, Javier Jiménez-Díaz, Felipe Higuera-Sobrino, María C. Bastante-Díaz, Evangelina \\ Campos-Pérez de Madrid, Ángela Fernández-Trujillo, Laura Cano-Rosado y Ana M. Casas de Miguel \\ Unidad de Arritmias, Hospital General Universitario de Ciudad Real, Ciudad Real, España
}

\section{Resumen}

Objetivo: Describir el grado de satisfacción y el impacto sociolaboral y medioambiental de los pacientes de un programa de seguimiento remoto de marcapasos. Método: Estudio observacional prospectivo que incluyó 160 pacientes del programa de seguimiento remoto entre 2016 y 2017. Se pasó una encuesta de satisfacción a dichos pacientes y se cuantificó la disminución del $\mathrm{CO}_{2}$ emitido al reducir el número de visitas. Resultados: Los pacientes acudían acompañados (86\%) y en coche (66\%) la mayoría de las veces, y mostraron un grado de satisfacción «bueno o excelente» en un $96 \%$. Se estima un ahorro de emisión de $\mathrm{CO}_{2}$ de casi un $10 \%$ por ciclo de seguimiento. Conclusiones: El seguimiento remoto de marcapasos implantado en nuestra unidad de arritmias reduce el impacto sociolaboral, económico y medioambiental.

Palabras clave: Marcapasos. Arritmias. Dependencia funcional.

\section{Abstract}

Objective: To describe the degree of satisfaction and social, occupational and environmental impact of patients on our remote pacemaker monitoring programme run. Method: Prospective observational study including 160 patients on the remote pacemaker monitoring programme between 2016 and 2017. We handed out a satisfaction survey and quantified the decrease in $\mathrm{CO}_{2}$ emitted by reducing the number of visits. Results: The patients attended visits accompanied (86\%) and by car (66\%) most of the time. $96 \%$ of respondents said their degree of satisfaction was "good or excellent". We estimated a saving in $\mathrm{CO}_{2}$ emissions of almost $10 \%$ for each remote monitoring cycle. Conclusions: The remote monitoring of pacemakers implemented by our arrhythmia unit reduces the social, occupational, financial and environmental impact.

Keywords: Pacemakers. Arrhythmias. Functional dependence.

\section{Correspondencia:}

*Pedro Pérez-Díaz

E-mail: pedroperezdiaz61@gmail.com
Disponible en internet: 22-02-2022 Rev Colomb Cardiol. 2022;29(1):23-28 www.rccardiologia.com 0120-5633 / @ 2022 Sociedad Colombiana de Cardiología y Cirugía Cardiovascular. Publicado por Permanyer. Este es un artículo open access bajo la licencia CC BY-NC-ND (http://creativecommons.org/licenses/by-nc-nd/4.0/). 


\section{Introducción}

El incremento progresivo de la esperanza de vida que ha tenido lugar en España en los últimos años ha determinado que la edad media en el momento del implante de un marcapasos haya aumentado de 77 años en 2012 a 77.8 años en $2016^{1}$. El 50\% del total de primoimplantes de marcapasos corresponde a individuos mayores de 80 años. Esto, junto con la mejoría de los modos de estimulación y la aparición de nuevos algoritmos, ha determinado que el seguimiento de los pacientes portadores de dispositivos de electroestimulación haya experimentado una evolución hacia la complejidad en los últimos 15 años. Estas situaciones, aunadas a la periodicidad de seguimiento semestral (dispositivos bicamerales o tricamerales) o anual (dispositivos monocamerales) que recomiendan las guías de electroestimulación, conllevan una sobrecarga asistencial significativa ${ }^{2}$.

Las consultas específicas de seguimiento de marcapasos, que en muchos hospitales son manejadas enteramente por personal de enfermería cualificado, así como el seguimiento remoto de estos dispositivos de manera telemática, constituyen un modo de paliar esta situación. A pesar de ello, este método de seguimiento continúa suponiendo una carga de trabajo importante para los servicios de cardiología, así como consumo de tiempo y dinero. La literatura científica publicada al respecto determina que estos pacientes presentan una calidad de vida semejante a la de aquellos sometidos a seguimiento presencial hospitalario, con lo cual se reduce significativamente el número de visitas al hospital $^{3}$. Las empresas de dispositivos de estimulación cardiaca permanente han creado sistemas de control a distancia canalizados a través de enfermería del centro de salud de referencia ${ }^{4}$.

La utilidad de la monitorización remota de los dispositivos de resincronización cardiaca y de los desfibriladores implantables también ha sido ampliamente estudiada $^{5-9}$. Los estudios CONNECT ${ }^{10}$ y COMPASS $^{11}$, entre otros, han sugerido la utilidad de la monitorización remota de los marcapasos y los desfibriladores para la detección precoz de la fibrilación auricular de novo y otros parámetros de utilidad clínica, con el objetivo de optimizar el tratamiento médico, iniciar la anticoagulación y evitar la aparición de eventos cardiovasculares embólicos a largo plazo. El estudio IMPACT ${ }^{12}$ intentó analizar la prevención de ictus, embolia sistémica y sangrado mayor guiada por electrograma auricular en pacientes portadores de un dispositivo de resincronización cardiaca o un desfibrilador implantable, mediante seguimiento remoto; sin embargo, tuvo que ser suspendido por futilidad. El estudio TRUST ${ }^{13}$, por su parte, demostró una reducción en las visitas presenciales de los pacientes del $45 \%$ en el grupo sometido a seguimiento remoto, sin que esto se relacionase con un aumento significativo de la morbilidad.

El estudio EuroEco ${ }^{14}$ no consiguió demostrar una disminución del impacto económico neto en el seguimiento remoto de los desfibriladores al compararlo con el seguimiento presencial, si bien la población estudiada resultaba heterogénea, con la consiguiente posibilidad de sesgo de procedimiento. Por otro lado, Calò et al..$^{15}$ y Buchta et al. ${ }^{16}$ describieron una disminución significativa de los costos en pacientes con desfibrilador o resincronizador en el grupo incluido en un programa de monitorización remota, en comparación con el grupo de monitorización convencional. En España, el estudio PONIENTE ${ }^{17}$, realizado por la unidad de arritmias del Hospital de Poniente, en Almería, demostró que el seguimiento remoto de dispositivos de electroestimulación en pacientes mayores resultaba costo-efectivo respecto a la monitorización convencional, si bien es necesario ampliar el número de estudios para valorar la aplicación de este tipo de seguimientos en el sistema público de salud.

En el caso del área sanitaria de Ciudad Real, el control remoto de los marcapasos adquiere especial relevancia, ya que los pacientes se encuentran distribuidos en una amplia zona, alejados del hospital de referencia en muchos casos, debido a la poca densidad de población de dicha región (25.19 hab/km², muy inferior a la media de España, $92.47 \mathrm{hab} / \mathrm{km}^{2}$ ). Los pacientes acuden a nuestro hospital de forma presencial al mes del implante, a los 3 meses, a los 6 meses y al año. A continuación, se les da una carta para que la siguiente revisión (6 meses después) la realicen en su centro de salud de referencia (Miguelturra, Daimiel, Carrión, Torralba de Calatrava, etc.), mediante un monitor con cabezal, que permite transmitir toda la información relativa al marcapasos, con capacidad de envío vía internet a nuestro centro de referencia. Posteriormente, en caso de que todos los parámetros se encuentren dentro de los límites requeridos, se les notifica la normofunción del dispositivo por correo postal y se les pide que repitan la misma operación pasados otros 6 meses. En caso de normofunción del dispositivo, la siguiente revisión debe ser presencial a los 6 meses de la última (en total, 18 meses tras la última revisión presencial en el hospital). 
Con este sistema, se disminuye el número de desplazamientos del paciente (que en algunos casos supone hasta $90 \mathrm{~km}$ ), lo que se traduce en mayor autonomía del paciente, disminución del gasto de transporte sanitario y reducción del absentismo laboral de sus familiares y cuidadores principales. En caso de detectar alguna anomalía en cualquiera de los parámetros medidos en la transmisión por control remoto, se notifica mediante llamada telefónica al paciente, que debe acudir fuera de cita a consulta presencial para repetir la medición y tomar las medidas oportunas. En caso de agotamiento de la batería, las transmisiones aumentan la periodicidad en función de esta (cada 6 meses, cada 3 meses, mensual, etc.).

La «huella de carbono» se refiere la totalidad de gases de efecto invernadero emitidos por efecto directo o indirecto de un individuo, organización, evento o producto. Se mide en $\mathrm{kg}$ de dióxido de carbono $\left(\mathrm{CO}_{2}\right)$ y, en el caso de los desplazamientos en vehículos motorizados, depende del modelo del coche, el año de fabricación, el tipo de motor (diésel o gasolina) y la distancia del desplazamiento. El sector sociosanitario contribuye de forma significativa a las emisiones globales de $\mathrm{CO}_{2}$, debido fundamentalmente a los largos desplazamientos que deben realizar los pacientes en muchas ocasiones. La poca literatura científica relativa a dicho parámetro en pacientes que precisan atención médica de forma ambulante considera la «telemedicina» como una medida interesante para reducir las emisiones de $\mathrm{CO}_{2}$ a la atmósfera. Por esto, consideramos relevante describir y cuantificar en qué medida el seguimiento remoto de marcapasos puede ayudar a reducir no solo el impacto social, sino también el medioambiental, en los pacientes portadores de marcapasos.

\section{Método}

Este estudio pretende describir el grado de satisfacción de los pacientes que se encuentran bajo el sistema de seguimiento remoto de marcapasos en el área de Ciudad Real, así como sus implicaciones sociales. De la misma manera, se intenta cuantificar el impacto medioambiental positivo que supone dicho programa de seguimiento a través de un parámetro cuantitativo, como lo es la huella de carbono en dichos pacientes.

Para ello, se hizo un estudio observacional prospectivo que incluyó 160 pacientes a quienes se les asignó un seguimiento de manera remota en un hospital universitario entre enero de 2016 y enero de 2017. Se pasó una encuesta de satisfacción a dichos pacientes tras la revisión presencial, en la que se analizaban variables como tiempo de espera, atención y trato recibidos por parte del personal. Asimismo, se les interrogaba acerca de aspectos sociales relativos a dicho proceso, con variables como necesidad de acompañamiento por un familiar, modo de desplazamiento, kilómetros de desplazamiento, tiempo total invertido, situación laboral, gastos, etc. Posteriormente, se cuantificó la huella de carbono de cada uno de los individuos que se analizaron por cada viaje que realizaron, y se comparó con la que hubiesen emitido en caso de haber realizado todas las revisiones de forma presencial en la consulta. Finalmente, se exploró el ajuste a una distribución normal de las variables cuantitativas mediante las pruebas de Shapiro-Wilk y Kolmogórov-Smirnov para utilizar la media y la desviación típica o la mediana y la amplitud intercuartílica como medidas de centralización y dispersión, respectivamente, en el análisis descriptivo. Se utilizó el programa estadístico SPSS versión 18.0 (SPSS Inc., Chicago, Illinois, USA) para realizar el análisis descriptivo.

\section{Resultados}

Ninguna de las variables de evaluación cuantitativas que se usaron (tiempo invertido en seguimiento remoto, kilómetros recorridos, diferencia de huella de carbono por ciclo, etc.) se ajustó a una distribución normal, según las pruebas de Shapiro-Wilk y Kolmogórov-Smirnov, por lo que en estos casos se utilizaron la mediana y el rango intercuartílico como medidas de centralización y dispersión.

En cuanto a la revisión mediante seguimiento remoto en su centro de salud, la mayoría de los pacientes venían acompañados (68.9\%). Un 65.6\% acudían en coche particular, el $19.4 \%$ lo hacían en ambulancia, el $9.4 \%$ en autobús, el $3.8 \%$ en taxi, el $1.2 \%$ a pie y el $0.6 \%$ en moto (Fig. 1). En cuanto a los tipos de combustible, el coche utilizado para el desplazamiento al centro de salud fue diésel en un $75 \%$ de los casos, y de gasolina en un $25 \%$. La mediana de tiempo que supuso a los pacientes la visita a su centro de salud de referencia fue de $60 \pm 30$ minutos, la distancia recorrida (ida y vuelta) fue de $1.4 \pm 4.9 \mathrm{~km}$ (Tabla 1 ) y la cantidad de $\mathrm{CO}_{2}$ emitido a la atmósfera (huella de carbono) fue de $0.39 \pm 1.9 \mathrm{~kg}$. El tiempo desde la transmisión hasta la recepción del informe fue mayor de 14 días en un $45.3 \%$ de los pacientes, entre 8 y 14 días en un $32.7 \%$, de 4 a 7 días en el $9.4 \%$ y menos de 3 días en el 9.4\%; la transmisión telefónica del informe se realizó en un $3.1 \%$. Por su parte, los pacientes 


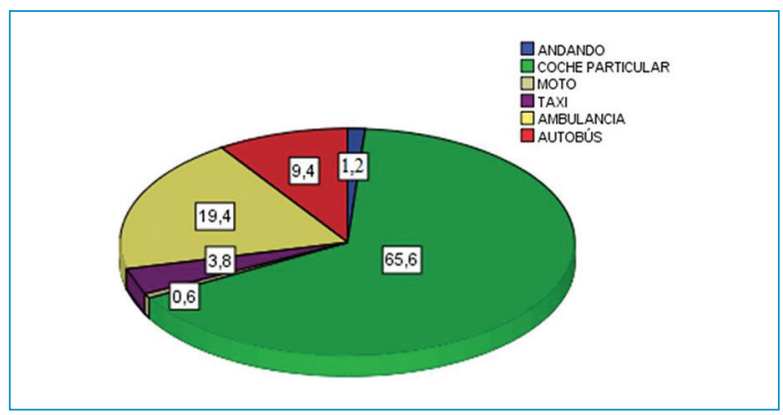

Figura 1. Modo de desplazamiento al centro de salud de referencia.

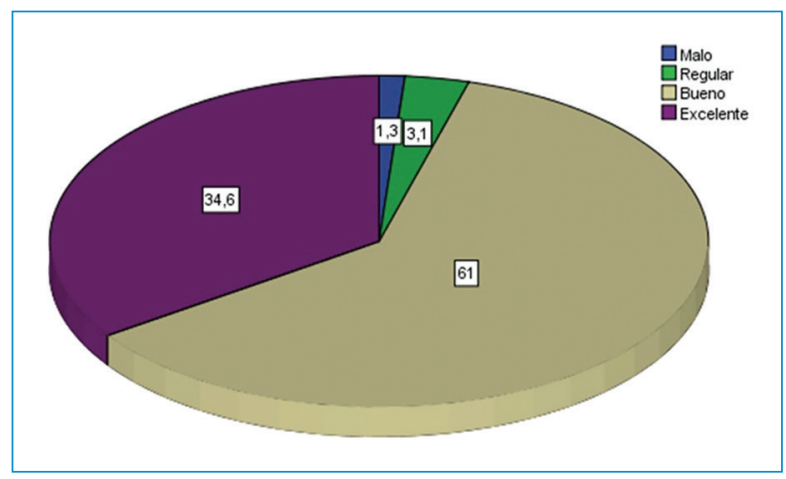

Figura 2. Grado de satisfacción con el seguimiento remoto.

mostraron un grado de satisfacción con el seguimiento remoto excelente en un $34.6 \%$, bueno en un $61 \%$, regular en un $3.1 \%$ y malo en un $1.3 \%$ (Fig. 2).

La encuesta de satisfacción de la visita presencial reveló que, al igual que en la visita en el centro de salud, en la revisión presencial la mayoría de los pacientes venían acompañados (85.6\%) y en coche particular $(66.2 \%)$, que era diésel en un $75 \%$ de los casos y de gasolina en el $25 \%$ restante. El $19.4 \%$ de los pacientes acudieron en ambulancia, el $8.8 \%$ en autobús, el $3.8 \%$ en taxi, el $1.2 \%$ andando y el $0.6 \%$ en moto (Fig. 3). La mediana de tiempo que suponía a los pacientes la visita al hospital fue de $150 \pm 120$ minutos, la mediana de la distancia recorrida (ida y vuelta) fue de $62 \pm 105.1 \mathrm{~km}$ (Tabla 2) y los gastos asociados al desplazamiento al hospital fueron de $5 \pm 10.5$ euros. La huella de carbono media emitida por los pacientes al acudir a la visita presencial fue de $124 \pm 197.2 \mathrm{~kg}$, tal que se estimó un ahorro de $15 \pm 33 \mathrm{~kg}$ de $\mathrm{CO}_{2}(9.6 \%)$ por paciente y ciclo anual de seguimiento remoto.

La mayoría de los pacientes que acudieron a nuestra consulta presentaban una situación de jubilación (90\%),

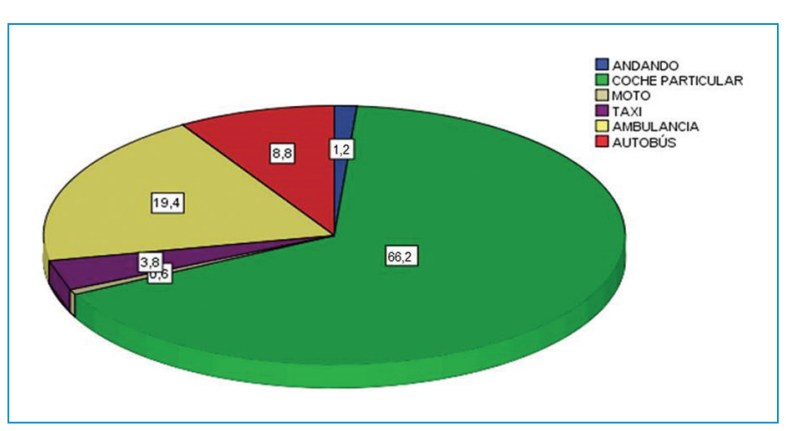

Figura 3. Modo de desplazamiento al hospital.

Tabla 1. Tiempos y distancias de desplazamiento al centro de salud de referencia

\begin{tabular}{|l|l|}
\hline Tiempo invertido & $60 \pm 30 \mathrm{~min}$ \\
\hline Distancia de desplazamiento & $1.4 \pm 4.9 \mathrm{~km}$ \\
\hline
\end{tabular}

Tabla 2. Tiempos y distancias de desplazamiento alhospital

\begin{tabular}{|l|l|}
\hline Tiempo invertido & $150 \pm 120 \mathrm{~min}$ \\
\hline Distancia de desplazamiento & $62 \pm 105.1 \mathrm{~km}$ \\
\hline
\end{tabular}

lo cual implicaba en muchos casos la necesidad de acompañante, el $54 \%$ de los cuales necesitaron ausentarse del trabajo para ello. Casi la mitad de los pacientes reconocía que el desplazamiento a la visita presencial afectaba mucho o muchísimo a su vida diaria y a la de su acompañante (46.5 y 52\%, respectivamente).

El tiempo de espera para ser atendido en la consulta fue de 30 a 45 minutos para el $23.6 \%$, de 15 a 30 minutos para el $18.6 \%$ y de 60 a 90 minutos para el $16.1 \%$. El trato recibido por parte del personal fue calificado como excelente en un $57.5 \%$ de los casos, bueno en un $41.9 \%$ y malo en un $0.6 \%$ (Fig. 4).

\section{Discusión}

El incremento de la esperanza de vida acontecido en España en los últimos años, junto con la evolución de los algoritmos de estimulación de los diferentes dispositivos, han determinado un impacto sociolaboral y económico muy importante derivado de las revisiones presenciales de dichos dispositivos. La baja densidad de población de la provincia de Ciudad Real, dentro de 


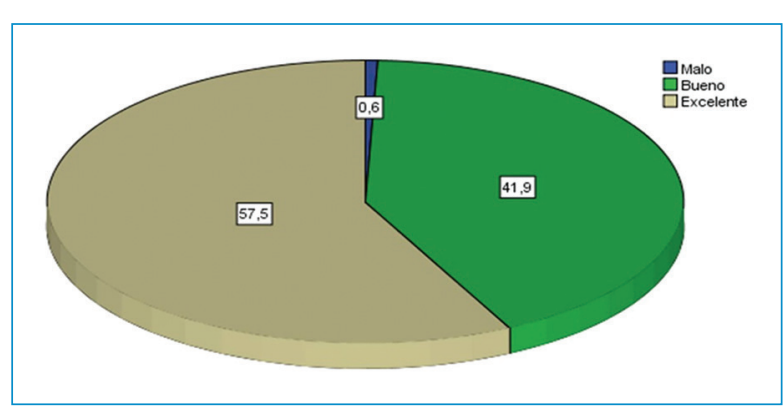

Figura 4. Trato recibido por el personal en consulta.

Castilla-La Mancha, determina que haya pacientes que tengan que desplazarse incluso hasta $90 \mathrm{~km}$ para realizar una revisión, la cual, según han demostrado numerosos estudios en la literatura, puede efectuarse con todas las garantías de seguridad y eficacia de manera telemática. Así mismo, más del $85 \%$ de los pacientes, según nuestros datos, necesitaban el acompañamiento de un familiar para acudir a la consulta hospitalaria, y de estos, el 54\% necesitó ausentarse del trabajo para ello, lo cual habla de la importante carga sociolaboral al sistema de trabajo español.

Los datos de este estudio muestran una disminución significativa del número de desplazamientos de los pacientes, que se relaciona con un grado de satisfacción muy positivo con el sistema de seguimiento remoto (el $95 \%$ de los pacientes consideraron dicho sistema de seguimiento como bueno o excelente en la encuesta de satisfacción). Este sistema contribuye a disminuir el gasto en transporte sanitario (el segundo modo de desplazamiento a la consulta por orden de frecuencia fue la ambulancia, 19.4\%) y a paliar el absentismo laboral de los cuidadores de los pacientes derivado del acompañamiento a las revisiones.

La dimensión medioambiental del sistema de seguimiento remoto implantado en Ciudad Real adquiere especial relevancia al objetivar que el $73 \%$ de los desplazamientos en vehículo particular se hacían con coches de motor diésel, y que la mediana de kilómetros que debían recorrer (ida y vuelta) para acudir a las revisiones del hospital fue de $62 \pm 105$. Puesto que el sector sociosanitario contribuye de forma significativa a las emisiones globales de $\mathrm{CO}_{2}$, se plantea que la huella de carbono (cantidad de $\mathrm{CO}_{2}$ emitido por efecto directo $\mathrm{o}$ indirecto de una determinada actividad) puede ser un marcador del impacto medioambiental del seguimiento remoto de los dispositivos de electroestimulación. De hecho, nuestro estudio ha demostrado un ahorro de $15 \pm 33 \mathrm{~kg}$ de $\mathrm{CO}_{2}$ (9.6\%) por paciente y ciclo anual de seguimiento remoto.

\section{Conclusiones}

El incremento de la edad media en el momento del implante de un marcapasos implica una sobrecarga asistencial de las consultas de revisión de dispositivos, así como un mayor desplazamiento de pacientes y familiares, que en muchos casos deben ausentarse de sus puestos de trabajo, un mayor gasto en transporte sanitario y, fundamentalmente, una menor autonomía del paciente. Los resultados de nuestro estudio sugieren que los sistemas de seguimiento remoto de marcapasos generan un impacto sanitario, social y laboral muy positivo, que se manifiesta tanto desde el punto de vista objetivo (mayor autonomía, menor tiempo invertido por consulta, menor distancia recorrida y disminución de la emisión de $\mathrm{CO}_{2}$ por ciclo de seguimiento en torno al 10\%) como subjetivo (percepción de satisfacción por parte de los pacientes y acompañantes).

\section{Financiamiento}

No se recibió ningún financiamiento externo para este trabajo.

\section{Conflicto de intereses}

Los autores declaran no presentar ningún conflicto de intereses.

\section{Responsabilidades éticas}

Protección de personas y animales. Los autores declaran que para esta investigación no se han realizado experimentos en seres humanos ni en animales.

Confidencialidad de los datos. Los autores declaran que han seguido los protocolos de su centro de trabajo sobre la publicación de datos de pacientes.

Derecho a la privacidad y consentimiento informado. Los autores han obtenido el consentimiento informado de los pacientes y/o sujetos referidos en el artículo. Este documento obra en poder del autor de correspondencia.

\section{Bibliografía}

\footnotetext{
1. Cano O, Pombo M, Fidalgo ML. XIV Informe Oficial de la Sección de Estimulación Cardiaca de la Sociedad Española de Cardiología (2016). Rev Esp Cardiol. 2017;70:1083-97.
} 
2. Brignole M, Auricchio A, Baron-Esquivias G, Bordachar $P$, Boriani $G$, Breithardl OA, et al. 2013 ESC guidelines on cardiac pacing and cardiac resynchronization therapy: the task force on cardiac pacing and resynchronization therapy of the European Society of Cardiology (ESC). Developed in collaboration with the European Heart Rhythm Association (EHRA). Eur Heart J. 2013;34:2281-329.

3. López-Villegas, Catalán-Matamoros D, Robles-Musso E, Peiro S. Efectividad comparativa del seguimiento remoto a personas con marcapasos cardíacos frente al convencional: calidad de vida a los 6 meses. Rev Esp Salud Pública. 2015;89:149-58.

4. Comoretto RI, Facchin D, Ghidina M, Proclemer A, Gregori D. Remote control improves quality of life in elderly pacemaker patients versus standard ambulatory-based follow-up. J Eval Clin Pract. 2017:23:681-9

5. Sáncho-Tello MJ, Ruiz-Mateas F, Fidalgo-Andrés ML. Avances en estimulación cardíaca. Rev Esp Cardiol. 2011;64(Supl 1):91-9.

6. Van Hemel NM. Remote monitoring of implanted cardiac devices: a plea for a nationwide exploration. Neth Heart J. 2009:17:434-7.

7. Burri H, Senouf D. Remote monitoring and follow-up of pacemakers and implantable cardioverter defibrillators. Europace. 2009;11:701-9.

8. Ricci R, Morichelli L, Santini M. Remote control of implanted devices through home monitoring technology improves detection and clinical management of atrial fibrillation. Europace. 2009;11:54-61.

9. Maciag A, Mitkowski P, Mazurek M, Kazmierczak J, Nowak K Grabowski M, et al. Patient perspective and safety of remote monitoring of implantable cardioverter-defibrillators in the Polish Nationwide Multicenter Registry: the Medtronic CareLink network evaluation. Kardiol Pol. 2020;78:1115-21.

10. Rossley GH, Boyle A, Vitense H, Chang Y, Mead RH. The CONNECT (Clinical Evaluation of Remote Notification to Reduce Time to Clinical Decision) trial: the value of wireless remote monitoring with automatic clinician alerts. J Am Coll Cardiol. 2011;57:1181-9.
11. Mabo P, Victor F, Bazin P, Ahres S, Babuty D, Da Costa A, et al. A randomized trial of long-term remote monitoring of pacemaker recipients (The COMPAS trial). Eur Heart J. 2012;33:1105-11.

12. Ip J, Waldo AL, Lip GY, Rothwell PM, Martin DT, Bersohn MM, et al IMPACT Investigators. Multicenter randomized study of anticoagulation guided by remote rhythm monitoring in patients with implantable cardioverter-defibrillator and CRT-D devices: rationale, design, and clinical characteristics of the initially enrolled cohort. The IMPACT study. Am Heart J. 2009;158:364-70.

13. Varma N, Epstein AE, Irimpen A, Schweikert R, Love C. Efficacy and safety of automatic remote monitoring for implantable cardioverter-defibrillator follow-up: The Lumos-T Safely Reduces Routine Office Device Follow-Up (TRUST) trial. Circulation. 2010;122:325-32.

14. Heidbuchel H, Hindricks G, Broadhurst P, Van Erven L, Fernández-Lozano I, Rivero-Ayerza M, et al. EuroEco (European Health Economic Trial on Home Monitoring in ICD Patients): a provider perspective in five European countries on costs and net financial impact of follow-up with or without remote monitoring. Eur Heart J. 2015;36:158-69.

15. Calò L, Gargaro A, De Ruvo E, Palozzi G, Sciarra L, Rebecchi M, et al. Economic impact of remote monitoring on ordinary follow-up of implantable cardioverter defibrillators as compared with conventional in-hospital visits. A single-center prospective and randomized study. J Interv Card Electrophysiol. 2013;37:69-78.

16. Buchta P, Tajstra M, Kurek A, Skrzypek M, Swietlinska M, Gadula-Gacek E, et al. The impact of remote monitoring of implanted cardioverter-defibrillator (ICD) and cardiac resynchronisation therapy device (CRT-D) patients on healthcare costs in the Silesian population: three-year follow-up. Kardiol Pol. 2017;75:573-80.

17. Bautista-Mesa RJ, López-Villegas A, Peiró S, Catalán-Matamoros D, Robles-Musso E, López-Liria R, et al. Long-term cost-utility analysis of remote monitoring of older patients with pacemakers: the PONIENTE study. BMC Geriatr. 2020;20:474. 